\title{
CITATION
}

Bayer, J. B., \& Hofstra, B. (2020). Toward Curation and Personality-Driven Social Networks. Nature Human Behaviour, 4, I23-125.

Published Version available at: https://doi.org/ I 0. I 038/s4 I 562-0 I 9-075 I-y

\section{Toward Curation and Personality-Driven Social Networks}

\author{
Joseph B. Bayer ${ }^{1,2}$ and Bas Hofstra ${ }^{3}$ \\ ' School of Communication, The Ohio State University \\ ${ }^{2}$ Translational Data Analytics Institute, The Ohio State University \\ ${ }^{3}$ Graduate School of Education, Stanford University
}

Whether or not social networks are significantly changing due to emergent technologies continues to be contested by researchers. Our understanding may advance by clarifying the cognitive mechanisms through which people curate their connections, along with the accompanying role of personality in shaping networks in the future.

Do humans have bigger or smaller social networks today? We reflect on the state of this research question and assert that an updated approach is needed to understand the effects of emergent technologies on network structure. Although the absolute changes in average network size are likely to remain elusive, recent perspectives converge on the idea that online technologies make it easier for individuals to shape-or curate- their social connections. Here we merge conflicting views and specify mechanisms through which curation technologies may impact personal network structure. Looking forward, we suggest personality will become more influential in network formation and maintenance when aided by technological levers. Consequently, curation technologies have the potential to increase differences in networks between types of people (for example, extroverts vs introverts) and thus generate new forms of social stratification, despite preserving a stable network size on average. The Comment concludes with empirical and theoretical implications, including the importance of attending to dispersion and examining the societal ramifications of personality-driven curation. 


\section{Changes to Personal Networks}

Many studies have sought to determine whether personal network size climbs or declines with Internet and mobile technologies. This question is crucial because the ways in which people construct and are constrained by their networks affect the accrual of social resources, as well as potential outcomes such as intergroup disparities (for example, job placement), social fragmentation (for example, racial segregation) and group polarization (for example, echo chambers) ${ }^{1,2}$. Nonetheless, our knowledge of the psychological factors that underpin personal networks remains scant and scattered, particularly when peering beyond the small core of intimate ties. And this challenge is further complicated with the ascent of novel network technologies.

Regrettably, past efforts do not lend themselves to straightforward answers to the principal question above. There are certainly more ways to reach personal ties today given the numerous channels available (for example, Facebook or Linkedln), not to mention the many sub-channels within platforms (for example, Newsfeeds or Messages). But paving more sidewalks is not akin to knowing more neighbours. Indeed, empirical research is unclear as to whether people today have smaller or larger networks than individuals of the past. Some research supports growth in average network size ${ }^{2}$, while other studies have suggested null or nebulous effects ${ }^{3,4}$.

The conflicting findings, in turn, have produced divergent viewpoints concerning the effects of new technologies on personal networks. One core perspective suggests that technologies cannot overcome the limitations of human cognition that would be required to maintain more friends ${ }^{4}$. Another fundamental view indicates that networks are becoming larger and more varied, highlighting ways in which humans have greater choice and control over their interactions ${ }^{2}$. That is, the former perspective emphasizes cognitive demands that limit having more 'real' friends, whereas the latter view stresses customizable levers that enable having more relationships. Altogether, one angle asserts that technologies do not alter the key network dimensions, while the other argues networks are being broadened and diversified.

The pair of classic perspectives thus come into conflict when answering the question of why personal networks are (or are not) in fact very different today. The challenge of identifying social network changes is compounded by a lack of specificity used to measure the technological behaviour in mind. This issue is significant given that other research areas (for example, digital inequality) have shown that measures of online access, adoption or features are insufficient to assess effects of technology; rather, individuals utilize the same tools in diverse ways. The takeaway is that average network changes evolving from institutional changes (for example, the genesis of online technologies) are surprisingly hard to capture.

In response to these obstacles, a revised approach is needed that clarifies how humans use technologies to shape their networks and considers a wider spectrum of effects. To bridge past perspectives, we propose shifting future research on technology effects toward network curation $^{5,6}$-i.e., how people prioritize communication with and attend to the communication of certain people. Whether or not humans maintain more friends in aggregate, curation tools may impact how different types of individuals build their networks. The following sections offer guidance on (i) identifying the cognitive mechanisms of curation and (ii) assessing the personality drivers of curation, along with their social consequences. 


\section{Mechanisms of Network Curation}

From MySpace's Top 8 to Facebook's Newsfeed, online platforms have unveiled a widening set of tools to maximize or minimize the salience of particular ties. A curation technology can be understood as any tool that facilitates the (re-)prioritization of certain relationships in addition to- or at the expense of-others. In their simplest forms, curation technologies increase the potential range of relationships visible by default use, as seen in phone contact lists or physical address books. Increasingly, however, network curation technologies encompass not only the abilities to (un)friend or (un)follow others, but also a growing toolkit to categorize key connections and prioritize their placement in automated streams of information. By centering technology behaviour on the process of curation itself, as opposed to the adoption or usage of particular devices or features, researchers will be better positioned to pinpoint network changes over time and space.

Importantly, future research should evaluate how technologies influence personal network structure (or lack thereof) by specifying the role of cognition. Past perspectives agree that relationship selection and maintenance are limited by cognitive demands, yet the precise pathways through which technologies matter for these processes remain ambiguous. As such, curation provides a lens through which to explicate and examine the cognitive mechanisms that underlie how people shape their network structure via technology. A cognitive approach to social network curation considers how technologies affect which friends come to mind, even if the objective amount of social behaviour is unchanged. Research is thus needed that assesses the degree to which social network cognition is altered through curation technologies, and two mechanisms to pursue are social network reflection and automation.

Specifically, curation technologies may influence network cognition by revising the relationship choice process with heightened reflection. Relationship maintenance has always involved the preference of certain ties over others, and online selection by no means overrides prior life experiences, contextual boundaries and social milieus that inherently narrow tie selection. Still, the control afforded by recent and rising channels gives humans a wider range of choices at each moment, during which they can elect to narrow or broaden their network scope. A range of tools contribute to the current flexibility of communication toolboxes ${ }^{7}$, and these are likely to be supplemented by more options (for example, virtual assistants, behavioural tracking, network visualization) that facilitate long-term network reflection.

Additionally, curation technologies may impact network cognition by automating the effortful work required to maintain ties. Whether one has to stroll down a street or swipe down a screen, relationship maintenance requires effortful engagement ${ }^{4}$. However, some research suggests individuals can groom relationships with more people through 'low-cost' one-to-many media'. In parallel, automated features such as feeds, reminders, notifications allow individuals to communicate with reduced cognitive effort. As such, newer technologies have the potential to offload cognitive steps in the maintenance process. For example, curation tools can streamline how people schedule intimate interactions, monitor life events and reactivate dormant relationships. With the right tools, one may not need to work so hard to keep up with Craig from college or Roman from high school; WhatsApp or WeChat will do it for you. 


\section{Personality-Driven Social Networks}

Emergent curation technologies thereby give humans more options in their social behaviour and may allow them to distribute cognitive resources in more versatile ways. This raises a secondary question about what types of people might adopt the tools that help rewire or offload the mental processing of social networks. Prior research demonstrates that personality is pivotal in explaining differences in personal network structure, as well as determining how people perceive and retrieve information about social networks ${ }^{8}$. For these reasons, personality traits are likely to guide which individuals adopt or avoid the cognitive tools available in a given culture. Even among the adopters, the discrete ways in which humans curate their social networks are likely to vary considerably-i.e., users will respond to the default and customizable settings of a given technology in distinct ways. In other words, the flexibility to reinforce certain relationships (as well as to ignore others) does not necessarily cause individuals to desire more friends, and it may not translate into more friends of equal quality.

Figure I | How curation might influence the distribution of network size

Use of curation technologies has the potential to generate a spreading effect on personal network sizes. Individuals who engage in a high degree of curation behaviour (pink) may gravitate to a wider range of social network sizes that reflect their social preferences, as compared to individuals who make less use of curation technologies (green and yellow).

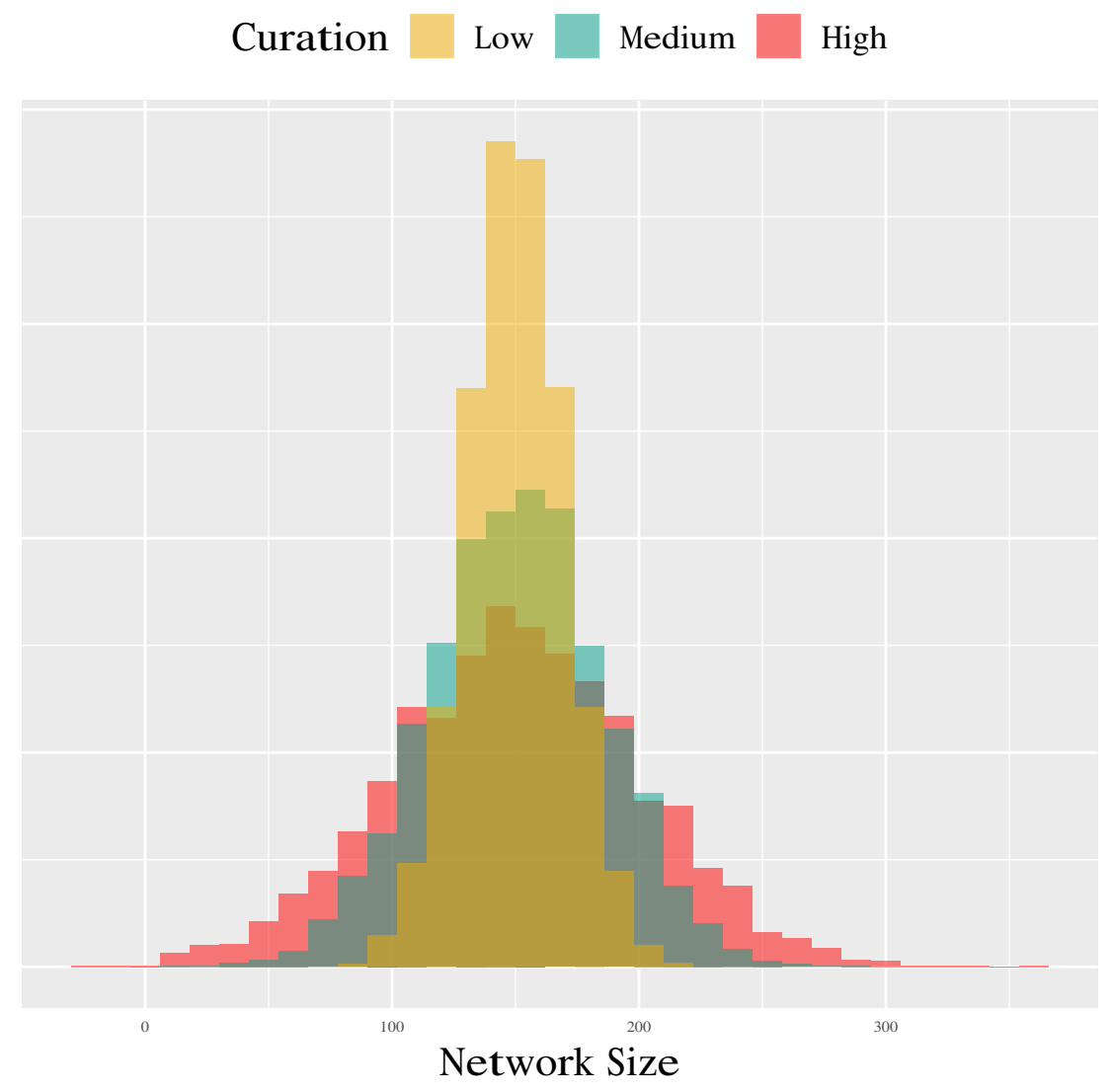


A focus on curation, therefore, raises broader questions about how emergent technologies widen or narrow the variation (i.e., dispersion or spread) in personal networks-not just how they cause changes to average network size. Despite the frequent focus on average network size in research on tech effects, network sizes vary considerably from one another', especially in the case of online networks ${ }^{4}$. As depicted in Fig. I, higher use of curation technologies (pink histogram) may increase the spread in the distribution of network sizes without shifting mean size (in this case, I50 friends for all histograms). Rather, this curation-to-variation hypothesis suggests individuals will engage in curation as a function of their network-relevant personality ${ }^{8}$. Consequently, research is needed to investigate how curation and personality jointly drive network structure. As humans are granted more mental tools to engage in curation, personality is poised to play a more pronounced role in shaping social networks.

Figure 2 | Impact of increasing curation non network size by personality type

As the level of curation behaviour rises, personality differences may facilitate higher variation in social network characteristics, independent of changes to average network size. The example below illustrates the potential spreading of network sizes through the interaction of curation and extraversion. Whereas extroverts (dashed green line) may maintain more friendships than in the past, introverts (dashed blue line) may reinforce fewer friendships-thus preserving the population average (white line).

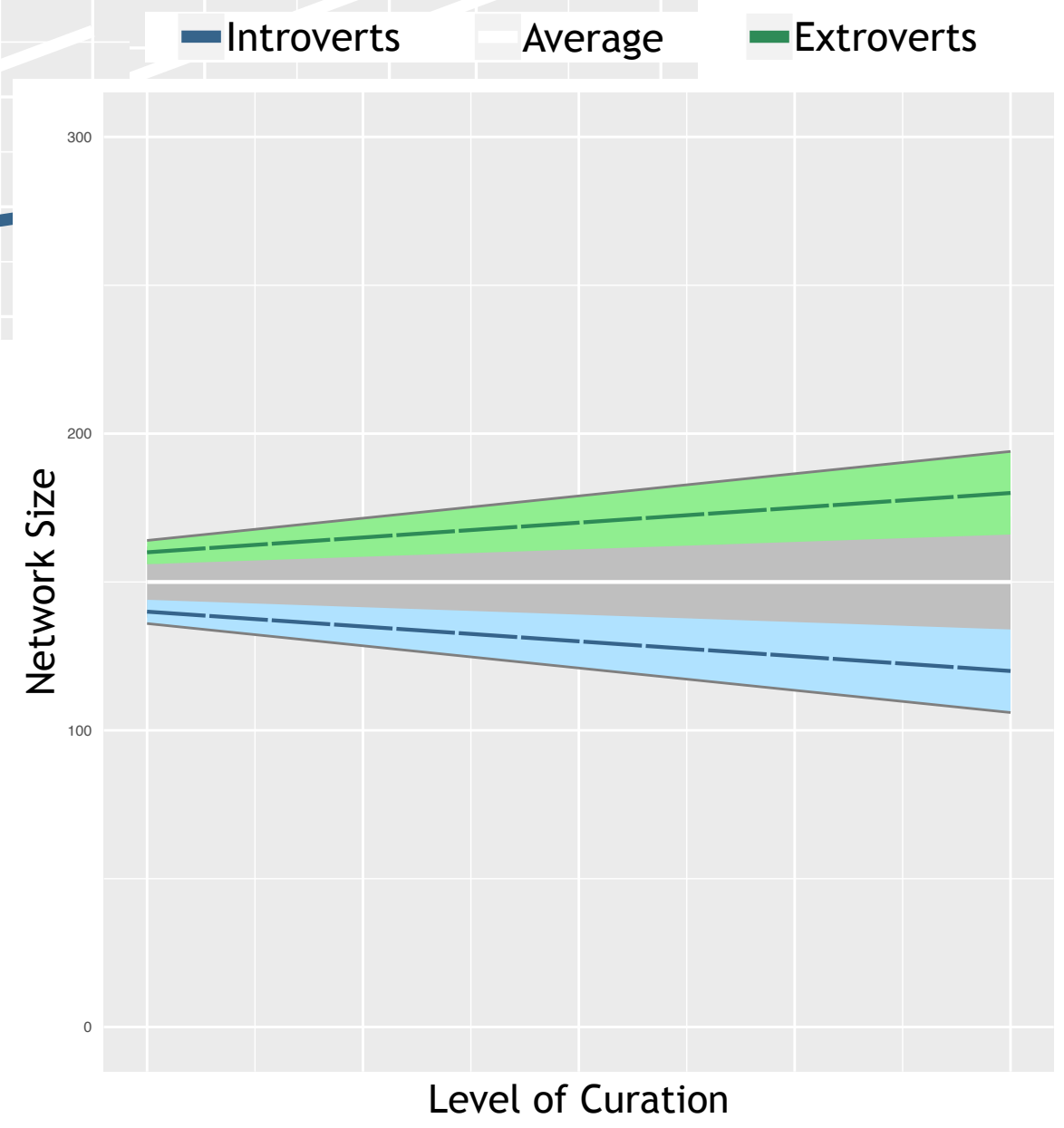


In this way, curation technologies have the potential to shift network size in divergent directions through the prism of personality. To illustrate this, we revisit the established positive relationship between extroversion and network size as an exemplar ${ }^{10}$. As displayed in Fig. 2, enhanced curation may lead extroverts (dashed green line) to choose to maintain more friends, and lead introverts (dashed blue line) to sustain fewer friends, without altering the mean (solid white line). Depending on the predispositions guiding an individual, curation capabilities hold the potential to increase, decrease or maintain their active network size. Thus, future networks may not be bigger in aggregate, but there is reason to expect bigger differences between them-i.e., spreading effects-as humans more fully exercise their social preferences.

Over the long-term, the ascent of personality-driven curation en masse also has the potential to influence collective, societal processes. In addition to curating the number of friendships that individuals maintain actively, individuals may curate other aspects of their networks too, such as prioritizing friends with certain personalities, families with certain morals, or cliques with certain norms. For instance, cognitive network curation may come to influence the level of personality homophily in communities, with certain network formations harmonizing and others conflicting with one another. These societal implications are comparable to recent concerns about elevated opinion homophily due to political newsfeed curation. Yet here we assert that social technologies have the potential to facilitate an array of novel stratification effects via the interaction of curation and personality.

\section{Conclusions}

This comment has sought to clarify and specify how emergent technologies relate to human network structure, offering a path for consolidating the contested literature on technology effects. We argue that past work has overlooked crucial mechanisms and outcomes, neglecting potential changes within subsets of individuals. Integrating past viewpoints, we chart a revised approach that targets the cognitive mechanisms underlying network curation. This perspective expands the research scope to consider whether technology causes humans to curate similar or different networks from one another. Hence, future work should test for differences in group variation (i.e., dispersion) in line with recent calls to attend to variation ${ }^{10}$.

In widening the research focus from technology usage to curation behaviour and from averages to dispersion, new findings may exhibit greater resilience across eras, locations and channels. Looking forward, we anticipate that social preferences will become more critical in evolving networks; or more tersely, personality matters more in personalized networks. As a consequence, curation technologies have the potential to widen the variation in personal network structure between types of individuals - and thus generate new forms of personality-driven stratification-whether or not future networks are 'bigger'. 


\section{References}

I. Wellman, B. Networks in the Global Village (Westview, 1999).

2. Rainie, L. \& Wellman, B. Networked: The New Social Operating System (MIT Press, 2012).

3. Brashears, M.E. \& Brashears, L.A. Emerging Trends in the Social and Behavioral Sciences I-I2 (Wiley, 20I5).

4. Dunbar, R. I. M. Trends Cogn. Sci. 22, 32-5I (20I8).

5. Davis, J. L. Inf. Commun. Soc. 20, 770-783 (2017).

6. Hogan, B. \& Quan-Haase, A. Bull. Sci. Technol. Soc. 30, 309-3I5 (20I0).

7. Hampton, K. Am. Behav. Sci. 60, I0I-I24 (2016).

8. Selden, M. \& Goodie, A. S. Soc. Networks 52, $81-99$ (20I8).

9. Roberts, S. G. B., Dunbar, R. I. M., Pollet, T. V. \& Kuppens, T. Soc. Networks 31, I38-146 (2009).

10. Ishiguro, I. Soc. Networks 44, 219-225 (2016). 